\title{
Circuit Model for Coplanar-Slotline Tees
}

\author{
Miquel Ribó, Associate Member, IEEE, and Lluís Pradell, Member, IEEE
}

\begin{abstract}
Coplanar-slotline tees have been used in a number of microwave circuits, although no complete models were available to explain their behavior. In this letter, a new "circuit-model" for a coplanar-slotline tee is presented. The model, based on the separation of coplanar waveguide (CPW) even and odd modes into two different ports, explains the tee's behavior even if CPW air bridges are not used and slotlines are loaded asymmetrically. The new model has been applied to the analysis of the input reflection coefficient of an offset-fed slotline antenna, showing an excellent agreement between simulation and measurement.
\end{abstract}

Index Terms-Circuit models, coplanar waveguides, slotline antennas, slotlines, transitions.

\section{INTRODUCTION}

$\mathbf{T}$ HE coplanar waveguide (CPW) is a multimode waveguide supporting two fundamental modes: coplanar even-mode (CEM), or coplanar-mode; and coplanar odd-mode (COM), or slotline-mode. The Slotline waveguide supports only one fundamental (slotline) mode. Coplanar-to-slotline transitions are widely used in uniplanar hybrid and monolithic microwave integrated circuits. A particular kind of CPW-to-slotline transition is the CPW-slotline tee shown in Fig. 1, in which either CPW slot is connected to a slotline. This transition is used in a number of circuits, such as slotline antennas [1]-[3], directional couplers [4], and hybrid rings and their applications (mixers and amplifiers [5]).

Whenever the tee's slotline sections are asymmetric or loaded asymmetrically, both modes, CEM and COM, are excited at the CPW section, and a detailed study of the $\mathrm{CPW}$-slotline tee behavior requires a circuit model in which both modes are taken into account. The tee's models available in the literature [3], [5] are not complete since they do not include the COM effect; in order to prevent the COM from propagating along the $\mathrm{CPW}$ section, either an air bridge connecting the CPW ground planes (at the transition plane) is used or the slotline ports are loaded symmetrically. In [6], the effect of air bridges on COM is numerically studied, but no circuit model is provided.

In this paper, a new four-port circuit model for CPW-slotline tees, such as those shown in Fig. 1, is proposed. In contrast to previous works [1]-[6], it studies a general case in which CPW's are asymmetric, air bridges are not connected, and the COM propagates along the CPW section. The model is based

Manuscript received February 3, 2000; revised March 27, 2000. This work was supported by the Spanish government under Grants 2FD97-0960-C05-05 and 2FD97-1769-C04-03 (CICYT- FEDER).

M. Ribó is with Enginyeria La Salle, Ramon Llull University, Barcelona 08022 Spain.

L. Pradell is with the Technical University of Catalunya, Barcelona 08034 Spain

Publisher Item Identifier S 1051-8207(00)04883-2.

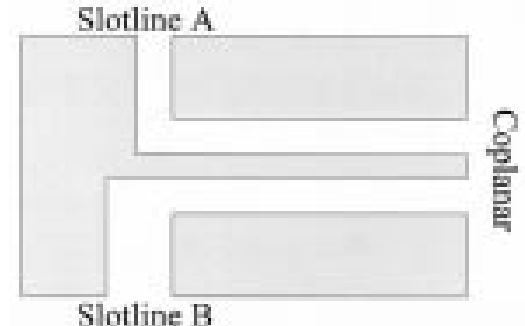

Fig. 1. Coplanar-slotline tee.
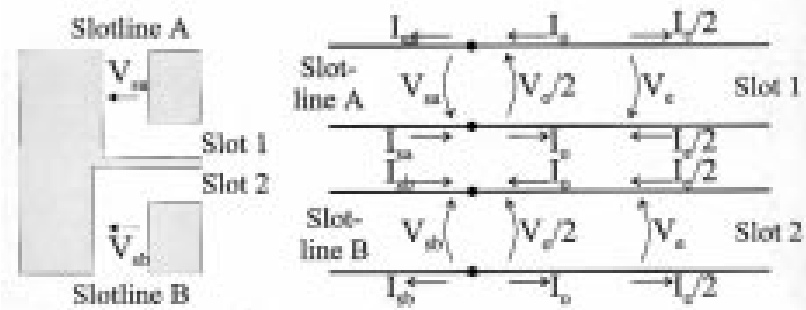

Fig. 2. Voltages and currents at the coplanar-slotline tee.

on the separation of modes (slotline, CEM and COM) into different ports, and therefore it provides a quantitative analysis of mode conversion (from slotline mode to CEM and COM, and vice versa) when the CPW section is loaded with general slotterminating planar structures that present different responses to each mode. Transitions with air bridges at the CPW section, such as those presented in [1], [2], [5], and [6], can also be analyzed as a particular case. Validation of the model is achieved through its application to the analysis of the input reflection coefficient, from $45 \mathrm{MHz}$ to $40 \mathrm{GHz}$, of an offset-fed slotline antenna.

\section{Model Derivation}

Consider the CPW-slotline tee shown in Fig. 1, performed by connecting a slotline to either CPW slot. Since the CPW can be excited asymmetrically by the slotline sections, both modes (CEM and COM) are present at the transition. Either CPW slot can be seen as a transmission line propagating both CEM and COM (see Fig. 2). The voltages and currents at the transition plane $\left(V_{o}, V_{e}, V_{\mathrm{sa}}, V_{\mathrm{sb}}, I_{o}, I_{e}, I_{\mathrm{sa}}\right.$, and $\left.I_{\mathrm{sb}}\right)$ are related by

$$
V_{\mathrm{sa}}=-\frac{V_{o}}{2}+V_{e}, \quad V_{\mathrm{sb}}=\frac{V_{o}}{2}+V_{e}
$$

and

$$
I_{\mathrm{sa}}=I_{o}-\frac{I_{e}}{2}, \quad I_{\mathrm{sb}}=-I_{\circ}-\frac{I_{e}}{2}
$$

where subscript $e$ stands for CEM, $o$ for COM, $s a$ for slotline $\mathrm{A}$, and $s b$ for slotline B. 


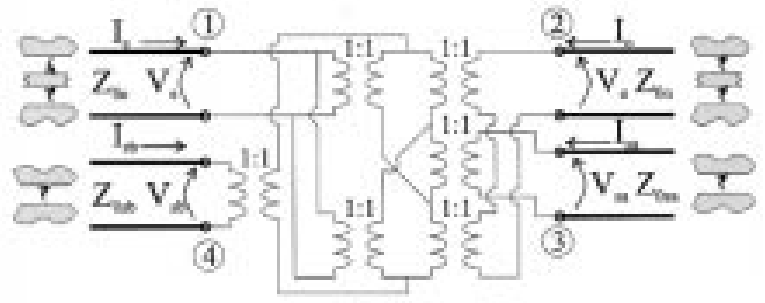

Fig. 3. Circuit-model proposed for a coplanar-slotline tee.

From (1) and (2), the following equation system is obtained for the normalized CEM, COM, and slotline incident and reflected waves at the transition plane, $a_{e}, a_{o}, a_{\mathrm{sa}}, a_{\mathrm{sb}}$ and $b_{e}, b_{o}, b_{\mathrm{sa}}, b_{\mathrm{sb}}$ respectively

$$
\left[\begin{array}{c}
b_{e} \\
b_{o} \\
b_{\mathrm{sa}} \\
b_{\mathrm{sb}}
\end{array}\right]=\frac{1}{3}\left[\begin{array}{cccc}
-1 & 0 & 2 & 2 \\
0 & 1 & -2 & 2 \\
2 & -2 & 0 & 1 \\
2 & 2 & 1 & 0
\end{array}\right] \cdot\left[\begin{array}{c}
a_{e} \\
a_{o} \\
a_{\mathrm{sa}} \\
a_{\mathrm{sb}}
\end{array}\right]
$$

where $a_{\alpha}=\left(V_{\alpha}+Z_{0} I_{\alpha}\right) \cdot\left(4 Z_{0}\right)^{-1 / 2}, b_{\alpha}=\left(V_{\alpha}-Z_{0} I_{\alpha}\right) \cdot$ $\left(4 Z_{0}\right)^{-1 / 2}(\alpha=e, o, s a, s b)$, and $Z_{0}$ is a real arbitrary impedance to which the normalized waves and the $4 \times 4$ $S$-parameter matrix are referred. The $S$-matrix obtained in (3) leads to the circuit model proposed in Fig. 3. The new model relevant features are as follows.

1) The three-port transition (two slotline ports and one multimode CPW port) is modeled as a four-port device that separates the slotline, CEM, and COM contributions into different single-mode ports, namely, two slotline ports (with characteristic impedances $Z_{0 \mathrm{sa}}$ and $Z_{0 \mathrm{sb}}$ ), one CPW port propagating only the COM (with characteristic impedance $Z_{0 \circ}$ ), and one CPW port propagating only the CEM (with characteristic impedance $Z_{0 e}$ ).

2) Therefore, planar structures that present different responses to each CPW mode (such as other slotline-to-CPW transitions and air bridges) can be easily connected by loading the ports (1) and (2) in Fig. 2 with appropriate impedances. Whenever a mode is cut off, its effect is also easily included by terminating the appropriate ports in Fig. 2 with reactive impedances.

3) It allows a quantitative analysis of the energy transfer from one mode to the others. In particular, whenever the slotline ports (3) and (4) in Fig. 3 are loaded symmetrically $\left(a_{\mathrm{sa}}=a_{\mathrm{sb}}\right)$, the COM is not generated, as seen from (3). Conversely, whenever the slotline ports (3) and (4) are loaded antisymmetrically $\left(a_{\mathrm{sa}}=-a_{\mathrm{sb}}\right)$, the CEM is not generated.

4) Since the model contains circuit elements, it is easily implementable in microwave computer-aided design.

5) The model is frequency independent.

\section{Model APPLICATION AND EXPERIMENTAL VALIDATION}

The proposed model has been applied to the analysis of the input reflection coefficient of an offset-fed slotline antenna [see Fig. 4(a)] fabricated on alumina with $\varepsilon_{r}=9.7$ and thickness $=0.635 \mathrm{~mm}$. Slotline and coplanar slot widths are $25 \mu \mathrm{m}$. The

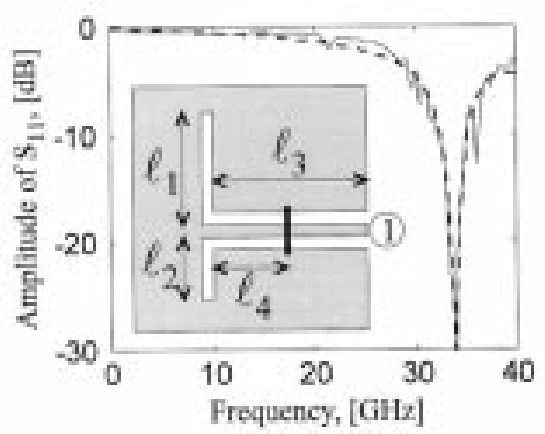

(a)

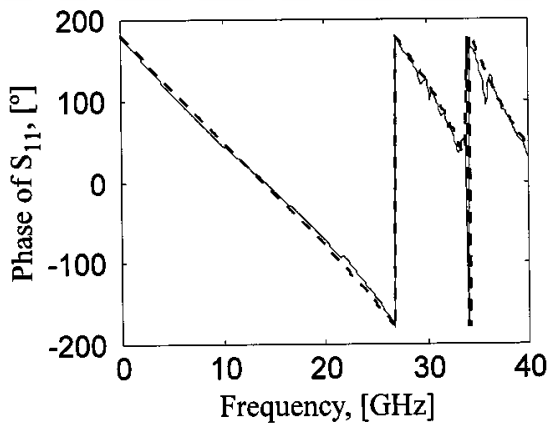

(b)

Fig. 4. Slotline antenna input reflection coefficient. Measurements are shown in continuous line and simulations in dashed line. (a) Magnitude of $S_{11}$ and antenna outline. (b) Phase of $S_{11}$.

coplanar central conductor width is $50 \mu \mathrm{m}$. The antenna consists of a CPW-slotline tee loaded with two asymmetric short-circuited slotline stubs $\left(\ell_{1}=1 \mathrm{~mm}, \ell_{2}=0.5 \mathrm{~mm}\right)$ and excited by a $\mathrm{CPW}$ section $\left(\ell_{3}=2 \mathrm{~mm}\right)$ propagating the CEM. Due to the asymmetry in the slotline stubs, the COM mode is generated at the transition and propagates backward along the CPW section a distance $\ell_{4}=1 \mathrm{~mm}$, until it is short-circuited by an air bridge. Fig. 4(a) and (b) compares its simulated input reflection coefficient (obtained using the model shown in Fig. 3) with its measured input reflection coefficient. Two discontinuity effects have been taken into account in the simulation: a small inductance (less than $10 \mathrm{pH}$ ), associated to the slotline short-circuits, and an inductance associated to the air bridge whose value is estimated analytically to be $50 \mathrm{pH}$. Small higher order mode effects at the transition plane have also been considered with no significant improvement of results. The excellent agreement between simulations and measurements (the model is accurately predicting the resonance frequency) demonstrates the model validity and applicability.

\section{CONCLUSION}

A new circuit model for CPW-slotline tees that separates the contributions of CPW modes into different ports has been proposed. The model overcomes limitations of previous models because it allows the analysis of a general case in which air bridges are not present and slotlines are asymmetrical or loaded asymmetrically. It has been successfully applied to the analysis of the input reflection coefficient of an offset-fed slotline antenna, 
showing an excellent agreement between simulation and experimental results.

\section{REFERENCES}

[1] J. Schoenberg, T. Mader, B. Shaw, and Z. B. Popovic, "Quasioptical antenna array amplifiers," in IEEE Microwave Theory Tech. Symp. Dig., 1995, pp. 605-608.

[2] S. Raman and G. M. Rebeiz, "Single- and dual-polarized slot-ring subharmonic receivers," in IEEE Microwave Theory Tech. Symp. Dig., vol. 2, 1997, pp. 565-568.
[3] A. Nesic, "Endfire slotline antennas excited by a coplanar waveguide," in IEEE Antennas and Propagation Society Int. Symp. Dig., 1991, pp. 700-702.

[4] S. Labonté and W. J. R. Hoefer, "Analysis and optimization of $E$-plane directional couplers," in IEEE Microwave Theory Tech. Symp. Dig., 1988, pp. 721-724.

[5] P.-C. Hsu, C. Nguyen, and M. Kintis, "Uniplanar broad-band push-pull FET amplifiers," IEEE Trans. Microwave Theory Tech., vol. 45, pp. 2150-2152, 1997.

[6] C.-Y. Lee, Y. Liu, and T. Itoh, "The effects of the coupled slotline mode and air-bridges on CPW and NLC waveguide discontinuities," IEEE Trans. Microwave Theory Tech., vol. 43, pp. 2759-2765, 1995. 